\title{
LA VOLCANOLOGÍA LATINOAMERICANA: ESTADO DE LA SITUACIÓN REGIONAL EN EL 2008 A PARTIR DEL ANÁLISIS DE SUS DEBILIDADES, AMENAZAS, FORTALEZAS Y OPORTUNIDADES
}

\section{LATIN AMERICAN VOLCANOLOGY: STATE OF THE REGIONAL SITUATION IN 2008 FROM THE ANALYSIS OF ITS WEAKNESSES, THREATS, STRENGHTS AND OPPORTUNITIES}

Hugo Delgado-Granados ${ }^{1, *}$, José G. Viramonte ${ }^{2} \&$ Guillermo E. Alvarado ${ }^{3}$

${ }^{1}$ Universidad Nacional Autónoma de México, Instituto de Geofísica, Departamento de Vulcanología, Circuito Exterior, C. U. Coyoacán 04510, México D. F. ${ }^{2}$ Instituto GEONORTE-INENCO CONICET y Universidad Nacional de Salta,

Av. Bolivia 5150, 4400 Salta, Argentina

${ }^{3}$ Área de Amenazas y Auscultación Sísmica y Volcánica, Instituto Costarricense de Electricidad. Apdo. 10032-1000, San José, Costa Rica.

Red Sismológica Nacional

*Autor para contacto: hugo@geofisica.unam.mx

(Recibido: 19/03/2012; aceptado: 26/11/2014)

\begin{abstract}
This paper shows a series of self-critical reflections of the reality of Latin American volcanology, from an analysis of its strengths, weaknesses, opportunities, and threats (SWOT) held in February 2008 in Antigua, Guatemala. Before the analysis, an account of the efforts made to unite the Latin American volcanological community is presented, up to the formation of an association (the Latin American Association of Volcanology, ALVO according to its Spanish acronym) that will strengthen and promote partnerships between Latin American volcanologists and their institutions for the benefit of society, particularly during eruptive crisis of the numerous active volcanoes in the Latin American region.

Keywords: SWOT, Latin American volcanology, ALVO, volcanic hazards, volcanic surveillance, emergency plans.
\end{abstract}

DELGADO-GRANADOS, H., VIRAMONTE, J. G. \& ALVARADO, G. E., 2015: La volcanología latinoamericana: Estado de la situación regional en el 2008 a partir del análisis de sus debilidades, amenazas, fortalezas y oportunidades.- Rev. Geol. Amér. Central, 52: 57-66, DOI: 10.15517/rgac.v0i52.18981 


\begin{abstract}
RESUMEN: El presente trabajo muestra una serie de reflexiones autocríticas de la realidad de la volcanología latinoamericana, a partir de un análisis de sus debilidades, amenazas, fortalezas y oportunidades (DAFO) llevada a cabo en febrero del 2008 en Antigua, Guatemala. Antes de llegar a este análisis, se hace una relatoría de los esfuerzos que se realizaron para unir a la comunidad volcanológica latinoamericana, hasta su culminación en la constitución de una asociación (Asociación Latinoamericana de Volcanología, ALVO) que permita fortalecer y promover los lazos de colaboración entre vulcanólogos de América Latina y sus instituciones para beneficio de la sociedad, particularmente en la atención de crisis eruptivas en los numerosos volcanes activos de la región latinoamericana.

Palabras clave: DAFO, volcanología latinoamericana, ALVO, peligros volcánicos, vigilancia volcánica, planes de emergencia.
\end{abstract}

\section{INTRODUCCIÓN}

La comunidad volcanológica latinoamericana se ha venido fortaleciendo en las décadas recientes y juega un papel muy importante en la cada vez más amplia comunidad volcanológica internacional. Muestra de ello son los congresos recientes de nivel internacional realizados en México (1997, 2007, 2008), Chile (2004), Ecuador (2006), Costa Rica (2010), así como actividades periódicas realizadas en diversos países tales como los cursos internacionales de Volcanología en Colima (México), Heredia (Costa Rica) y Salta (Argentina), además de una serie de cursos internacionales sobre diversos tópicos vulcanológicos realizados en México, Guatemala, El Salvador, Nicaragua, Costa Rica y Ecuador. Por otra parte se encuentra la activa participación de latinoamericanos dentro de la International Association of Volcanology and Chemistry of the Earth's Interior (IAVCEI).

En el pasado se ha reconocido una serie de limitaciones con la que cuenta la comunidad latinoamericana, como una alta deserción de los profesionales especializados en volcanología y ramas afines debido a mejores ofertas de trabajo, cambios y directrices geopolíticas en los mandos gerenciales, problemas con el idioma inglés, falta de recursos económicos, continuidad del soporte presupuestario, etc. (Alvarado et al., 1999; 2007). Sin embargo, a pesar de la continuada existencia de algunos de estos problemas, la comunidad sigue adelante en continuo progreso.

Alvarado et al. (1999) llegan a conclusiones interesantes dentro del ámbito de la volcanología latinoamericana (válidas para finales del siglo XX): a) Solamente 16 volcanes poseían suficientes sistemas de vigilancia para detectar alguna actividad anómala. Los países con mayores índices de volcanes monitoreados eran Colombia y Ecuador (58\% y 50\%, respectivamente). Sin embargo, observatorios que cumplen con los sistemas básicos de monitoreo por edificio volcánico vigilado, se encuentran en Guatemala y México, seguidos por Costa Rica y Nicaragua.

b) Durante la últimas dos décadas del siglo $\mathrm{XX}$, varios nuevos observatorios vulcanológicos y centros de investigación fueron establecidos en América Latina (por ejemplo en México, Guatemala, Nicaragua, Costa Rica, Colombia, Ecuador, Chile y Argentina). Sin embargo, muchos volcanes peligrosos de la región latinoamericana se mantenían aún pobremente estudiados, virtualmente poco o sin sistemas de monitoreo y representan una amenaza potencial ante futuras reactivaciones.

c) Las erupciones más destructivas de Latinoamérica y el Caribe: Monte Pelée (1902, Martinica), Santa María (1902, Guatemala), El Chichón (1982, México) y Nevado del Ruiz (1985, Colombia), representaron más del $75 \%$ del total de muertes por actividad volcánica del siglo XX.

d) Son pocos los volcanes en América Latina que hacia fines del siglo XX se encontraban relativamente bien estudiados desde el punto de vista vulcanológico: Nevado de Colima, Popocatépetl, Santa María, Cerro Negro, Arenal, Irazú, Turrialba, Nevado del Ruiz, Galeras, Puracé, Cotopaxi, Guagua Pichincha, Villarrica y Láscar.

e) Para 1999 se había estimado que cada vulcanólogo (en cualquiera de sus ramas) debería, 
en teoría, hacerse cargo de estudiar con todo detalle (desde la geología y mapas de peligrosidad, hasta todos los sistemas de vigilancia) de al menos 2 centros volcánicos de interés.

Esta situación, a lo largo de un cuarto de siglo, motivó a una comunidad creciente de vulcanólogos latinoamericanos, cada vez más consolidada, a meditar sobre formas o medios para agruparse, fortalecerse e intercambiar experiencias, ante un medio geológico, geopolítico, cultural e idiomático común.

En esta contribución se muestra el desarrollo de las ideas y evolución de la volcanología latinoamericana, más allá de los aspectos técnico-científicos, con una visión autocrítica de sus carencias y defectos, así como de sus ventajas y virtudes. Se hace una relatoría de intentos para organizar a la comunidad, así como ejercicios grupales que permitiesen a la comunidad volcanológica latinoamericana una organización y estructura que permita sobreponerse a sus carencias y deficiencias para poder mejorar sus condiciones de trabajo y aportar a la sociedad en su conjunto, un beneficio. Esta sucesión de eventos dio lugar a la formación de la Asociación Latinoamericana de Volcanología (ALVO) y en su proceso de formación, los ejercicios grupales en que participaron un grupo representativo de vulcanólogos latinoamericanos muestra las necesidades de agrupación de la comunidad vulcanológica, lo cual representa una enseñanza a otras comunidades en el ámbito internacional.

\section{PRIMEROS ESFUERZOS DE ORGANIZACIÓN DE LA COMUNIDAD LATINOAMERICANA}

\section{Kagoshima, Japón, 1988}

En la reunión de Kagoshima, Japón de 1988 se dieron cita vulcanólogos latinoamericanos (de México, Costa Rica, Colombia y Chile) para tratar temas diversos concernientes a la región latinoamericana, surgiendo la idea de unir a la comunidad vulcanológica latinoamericana y formar una asociación latinoamericana de volcanología.

\section{Nuevo México, EE. UU., 1989}

En Santa Fe, Nuevo México, durante una Asamblea General de la IAVCEI se llevó a cabo otra reunión y se mencionó la necesidad de crear una organización latinoamericana de Volcanología. Incluso se nombró preliminarmente como Asociación Latinoamericana de Volcanología (ALAVO), pero las ideas no fructificaron debido a la inexistente comunicación entre los asistentes después del evento.

\section{Tenerife, España, 2006}

Durante la reunión Garavolcán 2006, en Garachico, Tenerife, España, se dieron pasos firmes al planear la mejor forma de organizar a la comunidad volcanológica latinoamericana. En esta reunión de organización participaron vulcanólogos de México, Guatemala, El Salvador, Costa Rica, Chile y Argentina. Se acordó que se debía impulsar este proyecto con un carácter latinoamericano fundamentalmente. Los colegas españoles habían sugerido hacer una asociación que incluyera a los vulcanólogos ibéricos, pero a pesar de los grandes lazos de colaboración con los colegas españoles, se decidió constituir sólo una asociación para la región latinoamericana. Así, desde esta reunión de 2006, quedó establecido el carácter regional de la asociación.

\section{Antigua, Guatemala, 2008}

No obstante los deseos de organización, un factor importante era el conocimiento de la comunidad latinoamericana. Para ello era necesario el análisis de la situación imperante de la volcanología latinoamericana. La oportunidad se presentó durante la $1^{a}$ Reunión de la Red Iberoamericana de Volcanología, en la ciudad de La Antigua (Guatemala) del 19 al 22 de febrero del 2008, a la cual asistieron 23 profesionales procedentes de 12 de los 21 países de la comunidad Iberoamericana: México, Guatemala, El Salvador, Nicaragua, Costa Rica, Colombia, Ecuador, Perú, Argentina, Chile, Portugal y España. Esta reunión se aprovechó para 
organizar a la comunidad vulcanológica latinoamericana, aprovechando los resultados de un ejercicio de análisis regional.

El principal objetivo de esta reunión fue debatir sobre la implicación social más importante de la volcanología en Iberoamérica: la reducción del riesgo volcánico. El interés fundamental de este objetivo era conocer la situación y el estado actual de los esfuerzos que los distintos países miembros de la comunidad iberoamericana estaban materializando para la reducción del riesgo volcánico en la región. El análisis y la evaluación de estos esfuerzos para la reducción del riesgo volcánico se realizó a través de diferentes grupos de trabajo, tratando diversos aspectos relacionados con las acciones científico-técnicas destinadas a la reducción del riesgo volcánico que han sido recomendadas por la Organización de las Naciones Unidas para la Educación, la Ciencia y la Cultura (UNESCO) y la IAVCEI.

Para entender y mejorar las condiciones de la volcanología latinoamericana, era necesario realizar un primer análisis de su situación. Para ello, se muestra en la siguiente sección el análisis llevado a cabo en el año 2008 en la reunión de La Antigua, Guatemala.

Un segundo análisis de la situación actual de la volcanología latinoamericana se llevó a cabo posteriormente en Manizales, Colombia, en noviembre del año 2010. Los resultados de ese análisis no representan una actualización de los presentes resultados, sino un paso adicional para el entendimiento del "estado del arte" de la volcanología regional y, por ello, se publicarán de manera separada. En este trabajo solo se presentan los resultados del primer análisis.

\section{METODOLOGÍA}

Se realizó un análisis de la matriz DAFO (Debilidades, Amenazas, Fortalezas y Oportunidades), también conocido como FODA (en inglés es conocido como SWOT: Strengths, Weaknesses, Opportunities, Threats), herramienta de gran utilidad para entender y tomar decisiones, enfocado a las tres acciones destinadas para la reducción del riesgo y vigilancia volcánica: car- tografía de la peligrosidad volcánica, vigilancia volcánica y planes de emergencia.

El análisis DAFO es una metodología de estudio de la situación competitiva de una empresa, institución o ente dentro del mercado o medio, atendiendo a sus características internas para determinar sus debilidades, amenazas, fortalezas y oportunidades. Las debilidades y fortalezas son internas del ente bajo análisis; las amenazas y oportunidades se presentan en su entorno, es decir, son externas.

Durante la etapa de planeación estratégica y a partir del análisis DAFO, se debe poder contestar cada una de las siguientes preguntas: ¿Cómo se puede detener cada debilidad? ¿Cómo se puede defender de cada amenaza? ¿Cómo se puede aprovechar cada fortaleza? ¿Cómo se puede explotar cada oportunidad?

Esta herramienta fue creada a principios de la década de los setentas del siglo XX y produjo una revolución en el campo de la estrategia empresarial (Hill \& Westbrook, 1997; Menon et al., 1999). $\mathrm{Su}$ objetivo final es poder determinar las ventajas competitivas que tiene el ente bajo análisis y la estrategia genérica a emplear por ella que más le convenga en función de sus propias características y las del mercado o medio en que se encuentra.

Como productos se tuvieron los diagnósticos y estrategias sobre el programa para la reducción del riesgo volcánico en la comunidad iberoamericana por sectores territoriales de la comunidad (Sudamérica, Mesoamérica y Portugal-España). Aquí solo se presentan los resultados correspondientes a la comunidad latinoamericana.

El análisis consta de cuatro pasos: 1) Análisis externo (también conocido como "Modelo de las cinco fuerzas de Porter"), 2) Análisis interno, 3) Confección de la matriz DAFO, y 4) Determinación de la estrategia a emplear (Hill y Westbrook, 1997; Menon et al., 1999).

\section{DAFO LATINOAMERICANO}

A continuación se muestran los resultados obtenidos durante la ejecución del DAFO llevado a cabo con la participación de 23 vulcanólogos de 12 países en febrero del 2008. Un resumen de estos resultados pueden verse en el cuadro 1 . 


\section{Cartografía}

Debilidades:

-Escasez de recursos económicos, humanos e infraestructura.

-Falta de coordinación institucional, interinstitucional e intergubernamental.

-Falta de una metodología sistemática unificada.

-Falta de un esquema de prioridades.

-Disponibilidad de información en forma organizada y centralizada.

-Falta de incentivos para asegurar la permanencia del personal

-Falta de planes preconcebidos de investigación y vigilancia durante crisis volcánicas.

\section{Amenazas:}

-Deficiencia de los sistemas civiles para mantener recursos humanos técnicos.

-Problemas conceptuales de población, autoridades y políticos.

-Falta de interés, conocimiento y visión de futuro en población, políticos y autoridades.

-Presencia de grupos de investigación externos en tiempos de crisis volcánicas sin coordinación con grupos locales.

\section{Fortalezas:}

-Experiencia y capacidad en trabajo geológico.

-Capacidad y nivel académico y técnico.

-Experiencia en elaboración de mapas de amenaza.

-Capacidad de producción y divulgación.

-Existencia de infraestructura y recursos (laboratorios analíticos, instrumental, computadoras, software, internet).

-Existencia de lazos de cooperación internacional e interinstitucional.

-Capacidad de gestión.

\section{Oportunidades:}

-Ocurrencia de crisis de origen volcánico para plantear planes de trabajo preconcebidos.

-Aprovechar el sistema educativo y de comunicación para orientarlo a la divulgación y enseñanza de la cartografía.

-Existencia de convenios y tratados internacionales.

-Existencia de programas y grupos de investigación nacionales e internacionales.
-Establecimiento de investigación con expertos regionales e internacionales.

\section{Vigilancia}

\section{Debilidades:}

-Escasez de recursos económicos (adquisición y mantenimiento de equipo), humanos (actualización en la capacitación) e infraestructura (avance acelerado de la tecnología).

-Poder de convencimiento para invertir en la vigilancia.

-Líneas base de actividad volcánica incompletas o inexistentes en algunos volcanes.

-Conflictos entre grupos de trabajo e instituciones.

-Duplicidad de funciones.

-Insuficiencia de monitoreo de todos los volcanes activos de la región.

-Instrumentación y mantenimiento costosos o inalcanzables.

Amenazas:

-Grupos de trabajo en competencia durante crisis eruptivas.

-Percepción incorrecta de la magnitud de los efectos de la actividad volcánica por parte de población, políticos y autoridades.

-Crisis económicas nacionales y regionales que provocan recortes presupuestarios.

-Seguridad física del personal y del instrumental.

-Programas de trabajo internacionales que no toman en cuenta las necesidades locales.

\section{Fortalezas:}

-Existencia de recursos humanos con experiencia y capacitación en monitoreo básico.

-Existencia de infraestructura básica.

-Existencia de volcanes activos con potencial eruptivo.

-Existencia de bases de datos.

-Afinidades culturales y lingüísticas.

\section{Oportunidades:}

-Intercambio con grupos de investigación y entre grupos de trabajo.

-Reunión de expertos de la región.

-Existencia de programas nacionales e internacionales para adquisición de equipo.

-Creación de redes de vigilancia. 
Cuadro 1

Resumen de las debilidades, amenazas, fortalezas y oportunidades de la volcanología en América Latina

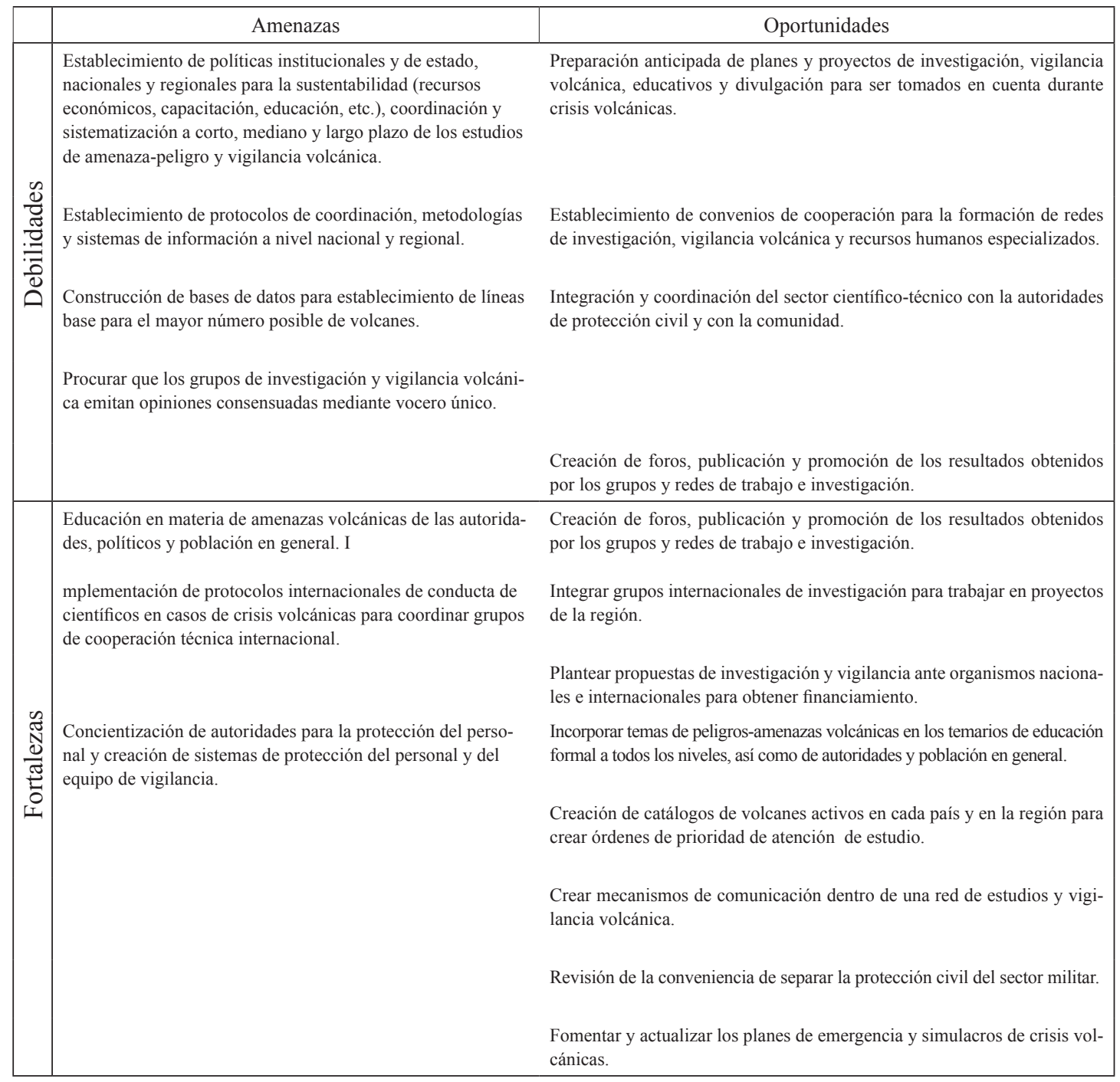

\section{Planes de emergencia}

\section{Debilidades:}

-Falta de aplicabilidad de la legislación en materia de protección civil.

-No existen planes de emergencia para todos los volcanes activos de la región.

-Falta de coordinación entre niveles de gobierno e interinstitucional.
-En la mayoría de los volcanes o países no se efectúan simulacros de emergencia volcánica.

-Falta de continuidad de planes y procesos de emergencia

-Fallas en el sistema de comunicación entre el sector científico técnico y las autoridades de protección civil. 
Amenazas:

-Cambios de régimen gubernamental.

-Pérdida de credibilidad.

-Competencia entre grupos de trabajo.

-Crisis volcánicas en momentos y situaciones de descoordinación

\section{Fortalezas:}

-Existencia de legislación en materia de protección civil.

-Existencia de personal capacitado.

-Capacidad de convocatoria en situaciones de crisis volcánicas.

-Existencia de planes de reacción y emergencia.

-Sistema de comunicación entre el sector científico técnico y las autoridades de protección civil.

\section{Oportunidades:}

-Separación de la protección civil del sector militar. -Intercambio de experiencias a nivel regional.

-Organización y capacitación a nivel comunitario. -Sistemas de reacción regional e internacional.

-Existencia de ONG.

\section{LA ASOCIACIÓN LATINOAMERICANA DE VOLCANOLOGÍA (ALVO)}

Luego de las reuniones antecedentes y tomando en cuenta los resultados del análisis DAFO del 2008, se consideró necesario que, para poder enfrentar exitosamente las debilidades y amenazas que se hicieron evidentes en el análisis, era necesario aprovechar las oportunidades mediante el aprovechamiento de las fortalezas. Esto sería posible mediante la colaboración de la comunidad latinoamericana y para ello, se hacía necesario el establecimiento de un ente formal regional. Para su constitución se realizaron diferentes discusiones en varios foros, como se menciona a continuación.

\section{Antigua, Guatemala, 2008}

Durante la $1^{a}$ Reunión de la Red Iberoamericana de Volcanología, en la ciudad de La Antigua (Guatemala) se contempló impulsar la creación de una Red Iberoamericana de Volcanología, o la Asociación Latinoamericana de Volcanología, o ambas, que la conformarían instituciones de la comunidad que tienen entre sus funciones y deseos la reducción del riesgo volcánico en los países miembros de la comunidad Iberoamericana. Finalmente, se decidió formar una asociación latinoamericana que permitiese la interacción entre individuos de la comunidad latinoamericana, mientras que la red involucraría instituciones. La agrupación que se planeó constituir como una asociación científica-técnica-educativa-cultural, tendría como objeto principal fomentar el intercambio de conocimientos y experiencias entre las personas que trabajan en el campo de la volcanología y de la gestión del riesgo volcánico así como la potenciación de la cooperación como método de trabajo. En un principio, su misión no sería obtener un resultado tangible, ya sea producto, proceso o servicio, sino crear un marco de colaboración en el trabajo que permita que en el futuro puedan surgir acciones conjuntas fundamentalmente destinadas al fomento de la volcanología y a una mejor gestión del riesgo volcánico. Dada la naturaleza multidisciplinaria de la gestión del riesgo volcánico, la entidad propuesta pretende ser un punto de encuentro de personal individual de instituciones de diversa naturaleza para debatir e impulsar estrategias para la reducción del riesgo volcánico en Iberoamérica: observatorios vulcanológicos, universidades, centros de investigación y tecnológicos, servicios de protección civil, sociedades científicas, empresas tecnológicas, colegios profesionales de geólogos, sociólogos, psicólogos, economistas, arquitectos, etc.

Dado que uno de los fines de la asociación es mejorar y optimizar los esfuerzos que la sociedad en su conjunto debe materializar para realizar una buena gestión del riesgo volcánico y este es un ejercicio de naturaleza multidisciplinaria que involucra a geocientíficos, autoridades, educadores, sociólogos, profesionales de la comunicación, psicólogos, responsables de emergencias, economistas y responsables de planificación urbana, los 
promotores de la Asociación Latinoamericana de Volcanología, desean que los asociados a esta red sean de instituciones y organizaciones públicas o privadas relacionadas no solo con la volcanología en general, sino también aquellas que tienen un papel que desempeñar en el ejercicio de la gestión del riesgo volcánico.

\section{Tenerife, España, 2010}

Durante la reunión Cities on Volcanoes en Santa Cruz de Tenerife, participó un grupo nutrido de vulcanólogos latinoamericanos de México, Nicaragua, Costa Rica, Puerto Rico, Colombia, Ecuador, Chile y Argentina. Se acordó la constitución definitiva de la ALVO con fecha de lanzamiento en noviembre de 2010 en Manizales, Colombia.

\section{Foz de Iguazú, Brasil, 2010}

En la reunión de la American Geophyisical Union en Foz de Iguazú participaron vulcanólogos latinoamericanos de México, Colombia, Ecuador, Chile y Argentina. Se confirmó que el lanzamiento de la ALVO sería durante la reunión de Manizales, Colombia y se discutieron los detalles de la formación de la asociación.

\section{Manizales, Colombia, 7 de noviembre del 2010}

En esta reunión y con la presencia del presidente de la IAVCEI en ese tiempo, Prof. Setsuya Nakada, se lanzó la ALVO. Participó un numeroso grupo de vulcanólogos de Latinoamérica: Argentina, Chile, Colombia, Costa Rica, Ecuador, El Salvador, Guatemala, México, Nicaragua, Panamá, y Perú. En esta ocasión se formalizó ante notario público la constitución de la asociación y sus estatutos. Asimismo se eligieron sus autoridades.

Los principales objetivos de la ALVO son fortalecer y promover los lazos de amistad y colaboración entre vulcanólogos de América Latina. Asimismo promueve la colaboración internacional dentro y fuera de la región latinoamericana.

\section{DISCUSIÓN}

La comunidad vulcanológica latinoamericana comparte aspectos que constituyen una gran fortaleza regional. Ejemplo de estos aspectos son el idioma y la cercanía cultural de los vulcanólogos de los países latinoamericanos. No obstante las realidades políticas, económicas y sociales, que son particulares de cada país, varios aspectos fueron identificados de manera común.

Así, la ausencia de políticas institucionales para la sustentabilidad, coordinación y sistematización a corto, mediano y largo plazo de los estudios y vigilancia volcánica, es una de las amenazas que más preocupan a la mayoría de los vulcanólogos de la región, ya que esto redunda en el establecimiento de protocolos de coordinación, metodologías y sistemas de información a nivel nacional y regional. El resultado de esta problemática es el aumento de la vulnerabilidad de la población en caso de presentarse un evento eruptivo de gran escala en una región altamente poblada y expuesta.

La ausencia de estas políticas impacta directamente en el financiamiento y planeación de las actividades de estudio y vigilancia volcánica. Programas de trabajo de monitoreo no pueden ser concebidos sin una visión de largo plazo, ya que la vigilancia es una actividad que debe sostenerse a lo largo del tiempo, sin fechas límite o terminación, porque los fenómenos volcánicos no tienen patrones previsibles en la mayoría de los casos. Por otra parte, los magros recursos económicos en el mantenimiento de las redes de vigilancia y en la contratación de personal calificado, así como su capacitación, son un aspecto fundamental que no se contempla con la debida responsabilidad por parte de los administradores y políticos. Una red de vigilancia mal mantenida no sirve al momento de una crisis y los datos obtenidos por redes recuperadas, después de repararlas en medio de una crisis, podrían llegar demasiado tarde.

Con el fin de hacer evidentes los volcanes que requieren mayor atención para su estudio y vigilancia, es necesaria la construcción de bases de datos con el fin de crear listas de prioridad. De esta manera se podría planear mejor la realización 
de estudios y actividades de vigilancia, optimizando los recursos económicos dedicados a estas actividades.

Un problema grave que se vislumbra en la comunidad vulcanológica latinoamericana, es la competencia entre grupos de trabajo. Este no es un problema exclusivamente latinoamericano, pero afecta la credibilidad del sector científicotécnico frente a las autoridades y la población al emitirse opiniones diversas y contrarias durante un evento eruptivo. La comunidad vulcanológica ve necesario que los grupos que realizan investigación científica y vigilancia volcánica emitan opiniones consensuadas al atender situaciones de crisis eruptivas. No hacerlo demerita su labor y expone aún más a la población.

Este análisis de la situación de la volcanología en la región es de gran utilidad y puede serlo aún más, si las autoridades de protección civil, científicas y académicas dan lectura a esta autocrítica de parte de vulcanólogos de diferentes países que, aunque viven diferentes realidades y particularidades en sus actividades vulcanológicas diarias, diversos aspectos que se comparten permiten tener una visión de problemas y virtudes regionales que, de subsanarse, se podría lograr la superación de los diferentes grupos de trabajo de la región.

\section{CONCLUSIONES}

La comunidad vulcanológica latinoamericana ha crecido, aunque no al ritmo que requieren los problemas de los volcanes y su actividad en la región. Debido a la cercanía cultural y uso de un mismo idioma, la solución de problemas en el ámbito vulcanológico de la región latinoamericana es un aspecto que puede abordarse si se conocen las debilidades y amenazas que la comunidad vulcanológica enfrenta. Para resolver la problemática también es necesario reconocer las oportunidades que se presentan en la región y para aprovecharlas es necesario reconocer las fortalezas existentes. Al realizar el análisis DAFO para la región se hacen evidentes los aspectos que hay que abordar y cómo. La comunidad volcanológica reconoce sus características y en una actitud autocrítica establece la problemática y derrotero a seguir para resolverla. Un corolario de esta visión amplia de la situación de la volcanología latinoamericana, tal como se vislumbró en el año 2008, es la unión de la comunidad para enfrentar la problemática, compartiendo experiencias y estableciendo lazos de colaboración. En ese sentido se fue gestando la idea de organizarse a través de la formación de una asociación. La relatoría de formación de la Asociación Latinoamericana de Volcanología (ALVO) es necesaria para entender la evolución de la comunidad hasta la formación de la ALVO, teniendo en cuenta que la comunidad ha podido expresar sus debilidades, amenazas, fortalezas y oportunidades, a la vez que ha comenzado a abordar la problemática de manera conjunta y ordenada.

\section{AGRADECIMIENTOS}

Los autores reconocen el apoyo de los colegas y amigos españoles. En particular se agradece a los doctores Nemesio Pérez y Joan Martí. El Dr. Nemesio Pérez participó en la formación de la ALVO al proveer generosamente la ayuda necesaria y condiciones para el encuentro de los vulcanólogos latinoamericanos en reuniones internacionales cuyos fondos gestionó (para la reunión de Garavolcan 2006, en Garachico, Tenerife, España; la $1^{a}$ Reunión de la Red Iberoamericana de Volcanología, en la ciudad de La Antigua, Guatemala). La reunión de La Antigua fue promovida por la Fundación ITER y patrocinada por el Ministerio de Educación y Ciencia (España) a través de una acción complementaria del Plan Nacional de I+D+i contó con la colaboración de la Agencia Española de Cooperación Internacional (AECI), del Cabildo Insular de Tenerife (Fundación Canaria ITER), de la iniciativa comunitaria INTERREG IIIB Azores-MadeiraCanarias, y del Instituto Nacional de Sismología, Vulcanología, Meteorología e Hidrología de Guatemala. Por su parte, el Dr. Joan Martí ha dado impulso a la formación de la ALVO con sus comentarios a varios de los integrantes de la comunidad volcanológica latinoamericana. Aunque 
los resultados de otras acciones promovidas por el Dr. Martí serán publicados en forma separada, se hace un reconocimiento desde ahora a sus palabras y actitud de aliento.

Adicionalmente, se agradece a los colegas ibéricos que aportaron sus opiniones durante el análisis DAFO (Dr. Zilda França). También se agradece la participación de numerosos colegas latinoamericanos que, con su desinteresada colaboración y participación en el análisis DAFO y a lo largo de la constitución de la ALVO aportaron valiosos comentarios: Hugo Moreno, Marta Calvache, Martha Navarro, Felipe Aguilera, Mario Ruiz, Angélica Muñoz, Jerzy Mariño, Demetrio Escobar, Enrique Guevara, Gloria P. Cortés, María Martínez, Otoniel Matías y Samuel Bonis, entre otros. Agradecemos también las aportaciones y comentarios de muchos otros colegas que no son mencionados en esta lista. Se agradece a los lectores-árbitros anónimos y sus sugerencias.

\section{REFERENCIAS BIBLIOGRÁFICAS}

ALVARADO, G., ACEVEDO, A.P., MONSALVE, M.L., ESPÍNDOLA, J.M., GÓMEZ, D., HALL, M., NARANJO, J.A., PULGARÍN, B., RAIGOSA, J., SIGARÁN, C. \& VAN DER LAAT, R., 1999: El Desarrollo de la
Vulcanología en Latinoamérica en el Último

Cuarto del Siglo XX.- Rev. Geofísica, 51:

185-241.

ALVARADO, G.E., SOTO, G.J., PULLINGER, C.R., ESCOBAR, R., BONIS, S., ESCOBAR, D. \& NAVARRO, M., 2007:

Volcanica ctivity, hazards, and monitoring.En: BUNDSCHUH, J. \& ALVARADO, G.E. (eds): Central America: Geology, Resources and Hazards. Taylor \& Francis, Londres, 2: 1155-1188.

HILL, T. \& WESTBROOK, R., 1997: SWOT Analysis: It's Time for a Product Recall.Long Range Planning, 30(1): 46-52. DOI:10.1016/S0024-6301(96)00095-7.

MENON, A., BHARADWAJ, S.G., ADIDAM, P.T. \& EDISON, S.W., 1999: Antecedents and Consequences of Marketing Strategy Making.- J. Mark. (American Marketing Association), 63(2): 18-40, DOI:10.2307/1251943.JSTOR 1251943. 